Y. Miyahara

Nagoya Math. J.

Vol. 47 (1972), 111-144

\title{
ULTIMATE BOUNDEDNESS OF THE SYSTEMS GOVERNED BY STOCHASTIC DIFFERENTIAL EQUATIONS
}

\author{
YOSHIO MIYAHARA
}

\section{§1. Introduction.}

The stability of the systems given by ordinary differential equations or functional-differential equations has been studied by many mathematicians. The most powerful tool in this field seems to be the Liapunov's second method (see, for example [6]).

In this paper we study the stability of a system governed by a stochastic differential equation such that

$$
d X(t)=f(t, X(t)) d t+G(t, X(t)) d W(t),
$$

where $W(t)$ is a Wiener process. The system governed by such an equation arises in such a way that the stochastic noise perturbs a deterministic system. In order to investigate the equation (1), it is natural to generalize the method for deterministic cases, where the investigation heavily depends on the use of a Liapunov function.

W. M. Wonham called a system given by a temporary homogeneous stochastic differential equation to be weakly stable if the equation is non-degenerate and if the corresponding process is positive recurrent (cf. [5]). R. Z. Khas'minskii studied the stability at origin of the system which is given by (1) and which has a solution $X(t) \equiv 0$ with probability one. Being inspired by those results, we wish to treat more general cases. For this purpose we take the concept of ultimate boundedness of the moment of order $p>0$ to describe stability of the system (Definition 2.1, 2.2). This concept was first introduced by Zakai in [4]: We mention that this concept has come from that of ultimate boundedness in the theory of ordinary differential equation where the global stability is discussed. Many of our results develop the R. Z. Khas'minskii's results ([2], [3]) for the ultimate boundedness problem.

Received November 24, 1971. 
In $\S 2$ we give definitions of the stability and we prove lemmas which play the basic role in the proofs of the theorems in $\S 3$ and $\S 4$. Lemma 2.1 is an improvement of Zakai's result [4, Theorem 1], however the proof requires a method used in [3].

In $\S 3$ we prove criterion theorems for the ultimate boundedness in terms of the Liapunov functions. Theorem 3.1 is our main theorem, which asserts that if a Liapunov function $V(t, x)$ of the system governed by (1) exists, then the system is $p$-th ultimately bounded. More precisely, if there exists a smooth function $V(t, x)$ which satisfies

$$
\begin{gathered}
V(t, x) \geqq c_{1}|x|^{p}-\alpha, \quad c_{1}, p, \alpha>0, \\
\mathscr{L} V(t \cdot x) \leqq-c_{2} V(t, x)+\beta, \quad c_{2}, \beta>0,
\end{gathered}
$$

where $\mathscr{L}$ is the generator of the Markov process corresponding to (1), then the system is $p$-th ultimately bounded. If $V(t, x)$ is of order $|x|^{p}$ for large $|x|$, then the system is exponentially $p$-th ultimately bounded. Theorem 3.3 gives a criterion for the system to be $p$-th ultimately bounded for all $p>0$.

Three examples illustrate how these theorems are used in order to check the ultimate boundedness of systems. In particular example 3.3 shows an interesting property that a system with a certain stable drift and a small diffusion is stable.

In $\S 4$ we treat converse theorems to the theorems in $\S 3$. We have not succeeded to establish complete converse theorems, but we have gotten many interesting results in this direction. For example, Corollary 4.1 shows that a temporary homogeneous system must have coefficients of order 1 for large $|x|$ in order that the system be exponentially $p$-th ultimately bounded.

In $\S 5$ we prove that the ultimate bounded process has a certain kind of recurrence property. This fact suggests us that the ultimate boundedness is a suitable concept to illustrate stability.

The author wishes to thank Professor H. Kunita for his advice in preparing the manuscript.

\section{§2. Definitions and lemmas.}

We consider a system governed by the stochastic differential equation

$$
d X(t)=f(t, X(t)) d t+G(t, X(t)) d W(t), \quad t \geqq 0,
$$


where $X(t)$ and $f(t, X(t))$ are $n$-vectors, $G(t, X(t))$ is an $n \times n$-matrix, and $W(t)$ is the $m$-dimensional standard Wiener process. The coefficients $f(t, x)$ and $G(t, x)$ are always assumed to satisfy

$$
\begin{gathered}
|f(t, x)|+|G(t, x)| \leq c(1+|x|) \\
|f(t, x)-f(t, y)|+|G(t, x)-G(t, y)| \leqq c|x-y|,
\end{gathered}
$$

where $|x|$ is the vector norm of $x$, and

$$
|G(t, x)|=\left(\sum_{i, j}\left|g_{i, j}\right|^{2}\right)^{1 / 2} \quad \text { with } \quad G(t, x)=\left(g_{i, j}(t, x)\right) .
$$

Under the assumptions $\left(A_{1}\right)$ and $\left(A_{2}\right)$, we are given the Markov process $X(t)$ which is the unique solution of (2.1) (see, [1, Chapter 8]).

DEFINITION 2.1. Let $p$ be a positive number. The process $X(t)$ which is given by (2.1) is said to be $p$-th ultimately bounded if there exists a constant $K$ such that for any $(t, x) \in[0, \infty) \times \mathrm{R}^{n}$ the following inequality holds :

$$
\varlimsup_{s \rightarrow \infty} M_{t, x}|X(s)|^{p} \leqq K,
$$

where $M_{t, x}$ stands for the conditional expectation under the initial condition $X(t)=x$.

DeFinition 2.2. The process $X(t)$ is said to be exponentially $p$-th ultimately bounded if there exist positive constants $K, c$ and $\alpha$ such that for any $(t, x) \in[0, \infty) \times R^{n}, s \geqq t$, the following inequality holds :

$$
M_{t, x}|X(s)|^{p} \leqq K+c|x|^{p} e^{-\alpha(s-t)} .
$$

Remark 1. If $X(t)$ is exponentially $p$-th ultimately bounded, then $X(t)$ is $p$-th ultimately bounded.

Remark 2. If $X(t)$ is (exponentially) $p$-th ultimately bounded for some $p$, then $X(t)$ is (exponentially) $q$-th ultimately bounded for $q \leqq p$.

DeFinition 2.3. The process $X(t)$ is said to be $q$-th ultimately unbounded if there exists a constant $K$ such that

$$
\lim _{s \rightarrow \infty} M_{t, x}|X(s)|^{q}=\infty \quad \text { for each }(t, x), \quad|x| \geqq K .
$$

Definition 2.4. The process $X(t)$ is said to be exponentially $q$-th 
ultimately unbounded if there exist positive constants $c, \alpha, K$ and $K^{\prime}$ such that the following inequality holds:

$$
M_{t, x}|X(s)|^{q} \geqq c|x|^{q} e^{\alpha(s-t)}-K^{\prime}, \quad \text { for }|x| \geqq K, \quad s \geqq t .
$$

Let $\mathscr{L}$ be the infinitesimal generator of the Markov process $X(t)$. Since $X(t)$ is the diffusion process which is the solution of $(2.1), \mathscr{L} V(t, x)$, $V$ being assumed to be smooth, is expressed in the form

$$
\mathscr{L} V(t, x)=\frac{\partial V}{\partial t}(t, x)+\sum_{i} f_{i}(t, x) \frac{\partial V}{\partial x_{i}}(t, x)+\frac{1}{2} \sum_{i, j} \sigma_{i j}(t, x) \frac{\partial^{2} V}{\partial x_{i} \partial x_{j}}(t, x),
$$

where $\left(\sigma_{i j}(t, x)\right)=G(t, x) \cdot G^{*}(t, x)$.

We now prove lemmas which will be used to prove the theorems in $\S 3$ and $\S 4$. The first one is a generalization of Theorem 4.1 in Khas'minskii [3] p. 113.

LEMMA 2.1. Let $X(t)$ be the process which is given by (2.1). Assume that there is a function $V(t, x)$ on $[0, \infty) \times R^{n}$ which satisfies the following conditions:

i) $V(t, x)$ is bounded from below, of $C^{2}$-class with respect to $x$ and of $C^{1}$-class with respect to $t$.

ii) $\mathscr{L} V(t, x) \leqq k_{1}+k_{2} V(t, x)$,

where $k_{1}$ and $k_{2}$ are some constants.

Then

iii) $\begin{aligned} M_{t, x} V(s, x(s)) & \leqq V(t, x) e^{k_{2}(s-t)}+\frac{\left|k_{1}\right|}{k_{2}}\left(e^{k_{2}(s-t)}-1\right), & \text { if } \quad k_{2} \neq 0, \\ & \leqq V(t, x)+\left|k_{1}\right|(s-t), & \text { if } \quad k_{2}=0,\end{aligned}$

for any $s \geq t$.

Proof. First we discuss the case where $k_{2}$ is not equal to zero. Set

$$
W(s, x) \equiv V(s, x) e^{-k_{2}(s-t)} .
$$

Let $\tau_{n}$ be the first exit time of $X(t)$ from $\left\{x \in R^{n} ;|x| \leqq n\right\}, n \geq 1$, and put $\tau_{n}(t) \equiv \min \left\{t, \tau_{n}\right\}$. Then $\tau_{n}(t)$ is a Markov time. Using the DynkinIto formula, we obtain

$$
M_{t, x} W\left(\tau_{n}(s), X\left(\tau_{n}(s)\right)\right)-W(t, x)=M_{t, x} \int_{t}^{\tau_{n}(s)} \mathscr{L} W(u, X(u)) d u,
$$


which is expressed, by (2.2), in the form

$$
M_{t, x} \int_{t}^{\tau_{n}(s)}\left\{-k_{2} V(u, X(u)) e^{-k_{2}(u-t)}+\mathscr{L} V(u, X(u)) e^{-k_{2}(u-t)}\right\} d u .
$$

By the use of (ii), this is bounded above by

$$
M_{t, x} \int_{t}^{\tau_{n}(s)} k_{1} e^{-k_{2}(u-t)} d u \leqq \int_{t}^{s}\left|k_{1}\right| e^{-k_{2}(u-t)} d u=\frac{\left|k_{1}\right|}{k_{2}}\left(1-e^{-k_{2}(s-t)}\right) .
$$

Since $W(u, x)$ is bounded from below on $[t, s] \times R^{n}$, we can apply the Fatou lemma to the left side of (2.3) to get

$$
M_{t, x} W(s, X(s)) \leqq W(t, x)+\frac{\left|k_{1}\right|}{k_{2}}\left(1-e^{-k_{2}(s-t)}\right)
$$

The inequality (iii) is derived immediately just by recalling that $W(s, x)$ $=V(s, x) e^{-k_{2}(s-t)}$.

In case $k_{2}=0$, we can easily prove

$$
M_{t, x} W\left(\tau_{n}(s), X\left(\tau_{n}(s)\right)\right) \leqq W(t, x)+\left|k_{1}\right|(s-t),
$$

from which (iii) follows.

LEMMA 2.2. Let $X(t)$ be as in Lemma 2.1. Let $p$ be any positive number, and let $h(x)$ be a function of $C^{2}$-class on $R^{n}$ such that

$$
h(x)=|x|^{p} \quad \text { for }|x| \geqq 1 .
$$

Then

$$
|\mathscr{L} h(x)| \leqq k_{3}+k_{4} h(x),
$$

where $k_{3}$ and $k_{4}$ are constants depending only on $h(x)$.

Proof. By the definition of the operator $\mathscr{L}$,

$$
\mathscr{L} h(x)=\sum_{i} f_{i}(t, x) \frac{\partial h}{\partial x_{i}}(x)+\frac{1}{2} \sum_{i, j} \sigma_{i j}(t, x) \frac{\partial^{2} h}{\partial x_{i} \partial x_{j}}(x) .
$$

Using $\left(\mathrm{A}_{1}\right)$ and (2.5), we have

$$
\begin{aligned}
\left|f_{i}(t, x)\right| & \leqq c(1+|x|), & \left|\sigma_{i j}(t, x)\right| & \leqq c(1+|x|), \\
\left|\frac{\partial h}{\partial x_{i}}(x)\right| & \leqq c^{\prime}\left(1+|x|^{p-1}\right), & & \frac{\partial^{2} h}{\partial x_{i} \partial x_{j}}(x) \mid \leqq c^{\prime}\left(1+|x|^{p-2}\right) .
\end{aligned}
$$

Conseqently $|\mathscr{L} h(x)| \leqq c^{\prime \prime}\left(1+|x|^{p}\right)$. By the assumption (2.5), we get 
$|x|^{p} \leqq c^{\prime \prime}+h(x)$. These two inequalities imply (2.6).

(Q.E.D.)

COROLLARY 2.1.

$$
M_{t, x}|X(s)|^{p} \leqq c\left(1+|x|^{p}\right) e^{k(s-t)}, \quad p \geqq 0 .
$$

Proof. We choose such $h(x)$ that

$$
|x|^{p}-2 \leqq h(x) \leqq|x|^{p}+1 .
$$

Applying Lemma 2.1 to $h(x)$, it holds that

$$
M_{t, x} h(X(s)) \leqq h(x) e^{k_{4}(s-t)}+\frac{k_{3}}{k_{4}}\left(e^{k_{4}(s-t)}-1\right)
$$

Therefore, we have

$$
\begin{aligned}
M_{t, x}|X(s)|^{p} & \leqq M_{t, x} h(X(s))+2 \leqq h(x) e^{k_{4}(s-t)}+2+\frac{k_{3}}{k_{4}} e^{k_{4}(s-t)} \\
& \leqq\left(|x|^{p}+1\right) e^{k_{4}(s-t)}+2+\frac{k_{3}}{k_{4}} e^{k_{4}(s-t)}
\end{aligned}
$$

The inequality (2.9) is nothing but the rephase of (2.7).

LEMMA 2.3. Let $V(t, x)$ be a function on $[0, \infty) \times R^{n}$ which satisfies the condition (i) in Lemma 2.1 and the following conditions:

(iv) $\mathscr{L} V(t, x) \geqq k_{3}+k_{4} V(t, x)$

where $k_{3}$ and $k_{4}$ are constants.

(v) $M_{t, x}|V(s, X(s))|, M_{t, x}\left|\frac{\partial V}{\partial t}(s, X(s))\right|, M_{t, x}\left|f_{i}(s, X(s)) \frac{\partial V}{\partial x_{i}}(s, X(s))\right|$ and $M_{t, x}\left|\sigma_{i j}(s, X(s)) \frac{\partial^{2} V}{\partial x_{i} \partial x_{j}}(s, X(s))\right|$ are bounded on any s-compact-subset of $[0, \infty)$ for any fixed $(t, x)$.

Then

$$
\begin{gathered}
M_{t, x} V(s, X(s)) \geqq V(t, x) e^{k_{4}(s-t)}+\frac{k_{3}}{k_{4}}\left(1-e^{k_{4}(s-t)}\right) \quad \text { if } k_{4} \neq 0 \\
M_{t, x} V(s, X(s)) \geqq V(t, x)+k_{3}(s-t) \quad \text { if } k_{4}=0 .
\end{gathered}
$$

Proof. First we discuss the case where $k_{4}$ is not equal to zero. Set

$$
W(s, x) \equiv V(s, x) e^{-k_{4}(s-t)} .
$$


By the assumption (v) of Lemma 2.3, we can apply the Dynkin-Ito formula to $W(s, x)$, and we have

$$
M_{t, x} W(s, X(s))-W(t, X)=M_{t, x} \int_{t}^{s} \mathscr{L} W(u, X(u)) d u
$$

Using the assumption (iv), we get

$$
\mathscr{L} W(u, X(u)) \geqq k_{3} e^{-k_{4}(s-t)} .
$$

We therefore have an inequality

$$
M_{t, x} W(s, X(s))-W(t, x) \geqq-\frac{k_{3}}{k_{4}}\left(e^{-k_{4}(s-t)}-1\right),
$$

which is equivalent to (2.10).

In case $k_{4}=0$, the proof is easy and is omitted.

COROLlaRY 2.2. Let $h(x)$ be the function defined in Lemma 2.2. Then we have

$$
M_{t, x} h(X(s)) \geqq h(x) e^{-k(s-t)}-C
$$

Proof. We can apply Lemma 2.3 to $h(x)$, since Corollary 2.1 and Lemma 2.2 assure the conditions (v) and (iv), respectively. The inequality (2.12) follows directly from (2.10).

(Q.E.D.)

\section{§3. Criterions for ultimate boundedness or unboundedness.}

First we establish a theorem which gives a sufficient condition for a process $X(t)$ determined by (2.1) in $\S 2$ to be $p$-th ultimately bounded.

THEOREM 3.1. Let $X(t)$ be the process which is given as the unique solution of (2.1) in $\S 2$, and let $p$ be a given positive number.

(A) If there exists a function $V(t, x)$ defined on $[0, \infty) \times R^{n}$ which satisfies the following conditions:

(i) $V(t, x)$ is of $C^{1}$-class w.r.t. $t$, and of $C^{2}$-class w.r.t. $x$.

(ii) $-\alpha_{1}+c_{1}|x|^{p} \leqq V(t, x)$,

where $c_{1}(>0)$ and $\alpha_{1}$ are constants.

(iii) $\mathscr{L} V(t, x) \leqq-c_{2} V(t, x)+\beta_{1}$,

where $\mathscr{L}$ is the same as defined in Lemma 2.1, $c_{2}(>0)$ and $\beta_{1}$ are constants, 
then the process $X(t)$ is $p$-th ultimately bounded.

(B) If $V(t, x)$ satisfies the conditions (i), (ii), (iii) in (A) and

(ii) $^{\prime} \quad V(t, x) \leqq c_{3}|x|^{p}+\alpha_{2}$,

where $c_{3}(>0)$ and $\alpha_{2}$ are constants,

then the process $X(t)$ is exponentially p-th ultimately bounded.

(C) If $V(t, x)$ satisfies (i), (ii), (iii) in (A) and in addition

(ii) $^{\prime \prime} \quad V(t, x) \leqq W(x) \quad$ for any $(t, x) \in[0, \infty) \times R^{n}$,

then for the function $T(t, x)$ given by

$$
T(t, x)=\inf \left\{\tau ; M_{t, x}|X(t+\tau+u)|^{p} \leqq K_{1}=K+1, \quad u \geqq 0\right\},
$$

it holds that

$$
T(t, x) \leqq c_{4} \log W(x)+c_{5} \quad \text { for any }(t, x),
$$

where $K$ is the constant which appeared in the definition of $p$-th ultimately boundedness, and $c_{4}$ and $c_{5}$ are some constants.

Proof. (A) By (i) and (iii), we can apply Lemma 2.1 to $V(t, x)$ by putting $k_{1}=\beta_{1}, k_{2}=-c_{2}$, so that we have

$$
\begin{aligned}
M_{t, x} V(s, X(s)) \leqq V(t, x) e^{-c_{2}(s-t)}+\frac{\beta_{1}}{c_{2}}\left(1-e^{-c_{2}(s-t)}\right) & \rightarrow \frac{\beta_{1}}{c_{2}} \\
& (\text { as } s \rightarrow \infty) .
\end{aligned}
$$

Using (ii) and (3.1), it is proved that

$$
M_{t, x}|X(s)|^{p} \leqq \frac{1}{c_{1}} M_{t, x} V(s, X(s))+\frac{\alpha_{1}}{c_{1}} \rightarrow \frac{\beta_{1}}{c_{1} c_{2}}+\frac{\alpha_{1}}{c_{1}} \quad(\text { as } s \rightarrow \infty) .
$$

With the choice of $K=\frac{\beta_{1}}{c_{1} c_{2}}+\frac{\alpha_{1}}{c_{1}}$, (3.2) shows that $X(t)$ satisfies the condition of Definition 2.1.

(B) Using (3.2), (3.1) and (ii)', we have

$$
M_{t, x}|X(s)|^{p} \leqq \frac{c_{3}}{c_{1}}|x|^{p} e^{-c_{2}(s-t)}+\left(K+\frac{\alpha_{2}}{c_{1}}\right) .
$$

This inequality shows that $X(t)$ is of exponential type.

(C) Using (3.1), (3.2) and (ii)", we have 


$$
M_{t, x}|X(s)|^{p} \leqq \frac{1}{c_{1}} W(x) e^{-c_{2}(s-t)}+K
$$

If we. define $\tau_{0}$ by

$$
\tau_{0}=\frac{1}{c_{2}} \log W(x)-\frac{1}{c_{2}} \log c_{1},
$$

then $T(t, x) \leqq \tau_{0}$, which implies

$$
T(t, x) \leqq c_{4} \log W(x)+c_{5} .
$$

(Q.E.D.)

A function $V(t, x)$ which appears in Theorem 3.1 is called a Liapunov function of the process $X(t)$.

Remark 3.1. The assertion (A) in Theorem 3.1 tells that we do not need to pay any attention about the integrability of $V(t, x)$. (cf. Zakai [4])

Remark 3.2. If the process $X(t)$ satisfies the conditions in (B), then $T(t, x)$ is estimated in the form

$$
T(t, x) \leqq d_{1} \log (1+|x|)+d_{2},
$$

where $d_{1}$ and $d_{2}$ are constants. While, if $e^{k|x|^{q}}+\alpha_{3}$ can be taken as $W(x)$ in $(\mathrm{C})$, then

$$
T(t, x) \leqq d_{3}|x|^{q}+d_{4}
$$

We then see a sufficient condition for the process $X(t)$ to be ultimately unbounded by means of Liapunov function.

THEOREM 3.2. Let $X(t)$ be the process which is given by (2.1) in $\S 2$, and let $q$ be a positive number.

(A) If there exists a function $V(t, x)$ defined on $[0, \infty) \times R^{n}$ which satisfies the following conditions:

(i) $V(t, x)$ is of $C^{1}$-class with respect to $t$ and of $C^{2}$-class with respect to $x$. Further $|V(t, x)|,\left|\frac{\partial V}{\partial t}\right|,\left|\frac{\partial V}{\partial x_{1}}\right|$ and $\left|\frac{\partial^{2} V}{\partial x_{i} \partial x_{j}}\right|$ are dominated by a certain polinomial of $x$.

(ii) $\lim _{\mid \overline{x \mid \rightarrow \infty}} V(t, x)=\infty$, and $V(t, x) \leqq c_{6}|x|^{q}+\alpha_{3}$, where $c_{6}$ and $\alpha_{3}$ are positive constants. 
(iii) $\mathscr{L} V(t, x) \geqq c_{7} V(t, x)-\beta_{2}$, where $c_{7}$ and $\beta_{2}$ are positive constants, then $X(t)$ is $q$-th ultimately unbounded.

(B) If $V(t, x)$ satisfies all conditions in (A) and further the following condition:

(ii) $^{\prime} \quad-\alpha_{4}+c_{8}|x|^{q} \leqq V(t, x)$, where $\alpha_{4}$ and $c_{8}$ are positive constants, then $X(t)$ is exponentially $q$-th ultimately unbounded.

Proof. The proof is nearly the same as that of Theorem 3.1, however in this case we need Lemma 2.3 in place of Lemma 2.1.

(A) By (i) and (iii), we can apply Lemma 2.3 to $V(t, x)$ by putting $k_{3}=-\beta_{2}, k_{4}=c_{7}$ :

$$
M_{t, x} V(s, X(s)) \geqq\left(V(t, x)-\frac{\beta_{3}}{c_{7}}\right) e^{c_{7}(s-t)}+\frac{\beta_{2}}{c^{7}} .
$$

Using the condition (ii) and (3.3), we have

$$
M_{t, x}|X(s)|^{q} \geqq\left(\frac{1}{c_{6}} V(t, x)-\frac{\beta_{3}}{c_{7}}\right) e^{c_{7}(s-t)}+\frac{\beta_{2}}{c_{6} c_{7}}-\frac{\alpha_{3}}{c_{6}} .
$$

In view of $\lim _{|\bar{x}| \rightarrow \infty} V(t, x)=\infty$, we see that (3.4) shows $q$-th ultimate unboundedness of the process $X(t)$.

(B) Using (3.4) and condition (ii)', we have

$$
M_{t, x}|X(s)|^{q} \geqq \frac{1}{c_{6}}\left(c_{8}|x|^{q}-\alpha_{4}-\frac{\alpha_{3}}{c_{7}}\right) e^{c_{7}(s-t)}+\frac{\beta_{2}}{c_{6} c_{7}}-\frac{\alpha_{3}}{c_{6}} .
$$

With the choice of $K$ such that $c_{8} K^{q}-\alpha_{4}-\frac{\alpha_{3}}{c_{7}}=\frac{1}{2} c_{8} K^{q}$, we know that (3.5) shows the exponentially $p$-th ultimate unboundedness of the process $X(t)$.

We now introduce a new concept which implies the $p$-th ultimate boundedness for all $p>0$.

DeFinition 3.1. We call $X(t) \omega$-th ultimately bounded if there exists a function $\varphi(x)=e^{a|x|^{b}}$ such that

$$
\varlimsup_{s \rightarrow \infty} M_{t, x} \varphi(X(s)) \leqq K \quad \text { for any }(t, x)
$$


where $a, b$ and $K$ are positive constants.

DEFINITION 3.2. We call $X(t)$ exponentially $\omega$-th ultimately bounded if there exists a functions $\varphi(x)=e^{a|x|^{b}}$ such that

$$
M_{t, x} \varphi(X(s)) \leqq c \varphi(x)^{r} e^{-\alpha(s-t)}+K^{\prime} \quad \text { for any }(t, x),
$$

where $a, b, c, r, \alpha$ and $K^{\prime}$ are positive constants. If, in particular, we can take $r=1$, then we call $X(t)$ strongly exponentially $\omega$-th ultimately bounded.

Remark 3.3. Evidently the following relations can be verified: strongly exponentially $\omega$-th ultimately bounded

$\Rightarrow$ exponentially $\omega$-th ultimately bounded

$\Rightarrow \omega$-th ultimately bounded

$\Rightarrow \infty$-th ultimately bounded (i.e. $p$-th ultimately bounded for any $p>0)$.

Later we will see that if the process $X(t)$ is exponentially $p$-th ultimately bounded for some $p>0$ and if it satisfies some additional assumptions, then $X(t)$ is strongly exponentially $\omega$-th ultimately bounded (cf. Corollary 4.2 in $\S 4$ ). But Example 3.2, as will be illustrated later, shows that the process $X(t)$ which is exponentially $p$-th ultimately bounded for some $p>0$ is not necessarily $\omega$-th ultimately bounded.

THEOREM 3.3. Let $X(t)$ be as in Theorem 3.1.

(A) Assume that there exists a Liapunov function $V(t, x)$ of $X(t)$ which satisfies the condition (i) and (iii) in Theorem 3.1 and the following condition:

(iv) $c_{9} e^{a|x|^{b}}-\alpha_{5} \leqq V(t, x)$, where $c_{9}>0, a>0, b>0$ and $\alpha_{5}$ are constants.

Then $X(t)$ is w-th ultimately bounded.

(B) If $V(t, x)$ satisfies the conditions (i), (iii) and (iv) in (A) and the following condition:

(iv) $)^{\prime} \quad V(t, x) \leqq c_{10} e^{a^{\prime}|x|^{b}}+\alpha_{6}$,

where $c_{10}>0, a^{\prime}>0$ and $\alpha_{6}$ are constants,

then $X(t)$ is exponentially $\omega$-th ultimately bounded. 
(B)' If $V(t, x)$ satisfies the conditions (i), (iii), (iv) and (iv)', and if we can choose $a^{\prime}$ in (iv)' to be equal to $a$ in (iv), then $X(t)$ is strongly exponentially $\omega$-th ultimately bounded.

Proof. (A) Using (iv) and (3.1) in the proof of Theorem 3.1, we have

$$
c_{9} M_{t, x} e^{a|X(s)|^{b}} \leqq V(t, x) e^{-c_{2}(s-t)}+\alpha_{5}+\frac{\beta_{1}}{c_{2}}\left(1-e^{-c_{2}(s-t)}\right)
$$

Hence we prove

$$
M_{t, x} e^{a|X(s)|^{b}} \leqq \frac{1}{c_{9}} V(t, x) e^{-c_{2}(s-t)}+\frac{\alpha_{5}}{c_{9}}+\frac{\beta_{1}}{c_{9} c_{2}} \underset{(s \rightarrow \infty)}{\longrightarrow} \frac{\alpha_{5}}{c_{9}}+\frac{\beta_{1}}{c_{9} c_{2}}
$$

The last inequality shows that $X(t)$ is $\omega$-th ultimately bounded.

(B) Using (3.6) and condition (iv)' we have

$$
M_{t, x} e^{a|X(s)|^{b}} \leqq \frac{c_{10}}{c_{9}} e^{a^{\prime}|x|^{b}} e^{-c_{2}(s-t)}+\frac{\alpha_{5}+\alpha_{6}}{c_{9}}+\frac{\beta_{1}}{c_{9} c_{2}}
$$

The inequality (3.7) shows that $X(t)$ is exponentially $\omega$-th ultimately bounded.

(B) $)^{\prime}$ With a particular choice of constants $a^{\prime}=a$ we obtain the result immediately.

(Q.E.D.)

The following examples serve to illustrate the role of Theorem 3.1, 3.2 and 3.3 in observing the stability of the system.

EXAMPLE 3.1. We consider an $n$-dimensional system

$$
d X(t)=A(t) X(t) d t+f(t, X(t)) d t+G(t, X(t)) d W(t), \quad t \geqq 0,
$$

where $A(t)$ is an $n \times n$ matrix, and $f(t, x)$ and $G(t, x)$ satisfy the conditions $\left(\mathrm{A}_{1}\right)$ and $\left(\mathrm{A}_{2}\right)$ in $\S 2$.

If the solution $x(t) \equiv 0$ of the corresponding deterministic system

$$
\frac{d x(t)}{d t}=A(t) x(t)
$$

is uniformly asymptotically stable, and if $f(t, x)$ and $G(t, x)$ satisfy

$$
\varlimsup_{|x| \rightarrow \infty} \frac{|f(t, x)|}{|x|}=\varlimsup_{|x| \rightarrow \infty} \frac{|G(t, x)|}{|x|}=0,
$$


then $X(t)$ is exponentially $p$-th ultimately bounded for any positive number $p$.

Proof. It is known that there is a Liapunov function $V(t, x)$ of the system (3.8)' which has the following properties:

a) $V(t, x)=(V(t) x, x)$,

where $V(t)$ is an $n \times n$-matrix,

b) $\mu|x|^{2} \leq V(t, x) \leq M|x|^{2}$, where $\mu$ and $M$ are positive constants,

c) there exists an $n \times n$-matrix-valued function $W(t)=\left(W_{i j}(t)\right)$ such that

$$
\begin{gathered}
\frac{d V(t, x)}{d t}=-(W(t) x, x), \quad \text { and } \\
\lambda|x|^{2} \leq(W(t) x, x) \leq \Lambda|x|^{2},
\end{gathered}
$$

where $\frac{d V}{d t}(t, x)$ is the derivative along the trajectory of (3.8) and $\lambda$ and $\Lambda$ are positive constants.

We now prove that this function $V(t, x)$ satisfies the assumptions of Theorem $3.1(B)$.

First we consider a particular case $p=2$. Then the conditions (i), (ii) and (ii)' are clearly satisfied. Let us examine the condition (iii).

$$
\mathscr{L} V(t, x)=-(W(t) x, x)+\sum_{i} \frac{\partial V(t, x)}{\partial x_{i}} f_{i}(t, x)+\frac{1}{2} \sum_{i, j} \sigma_{i j}(t, x) W_{i, j}(t),
$$

where $\sigma_{i j}(t, x)=G(t, x) \cdot G(t, x)^{*}$.

Since we have assumed (3.9), the second and the third terms of (3.10) are at most of order two for large $|x|$. Together with the property c) we have

$$
\mathscr{L} V(t, x) \leq-\frac{1}{2} \lambda|x|^{2}, \quad|x| \text { large enough, }
$$

which implies the condition (iii).

We then consider the case $p=2 m$. It can easily be seen, by the same way as the case $p=2$, that $V^{m}(t, x)$ satisfies the conditions in Theorem 3.1 (B). Thus, as we mentioned in Remark 2.2, $X(t)$ becomes exponentially $p$-th ultimately bounded for any $p \leqq 2 m$. Since $m$ can be 
taken as large as we wish, $X(t)$ is exponentially $p$-th ultimately bounded for any $p>0$.

(Q.E.D.)

EXAMPLE 3.2. We give an example which is exponentially $p$-th ultimately bounded for any $0<p<p_{0}$ and is exponentially $q$-th ultimately unbounded for any $q>p_{0}$ (it is, therefore, not $q$-th ultimately bounded).

For simplicity, we discuss the case $n=1$. Consider a linear system

$$
d X(t)=a X(t) d t+b X(t) d W(t)
$$

where $a(<0)$ and $b$ are constants. We take $V(t, x) \equiv h(x)$ as a Liapunov function of (3.11), where $h(x)$ is the function introduced in Lemma 2.2. Then we have

$$
\begin{aligned}
\mathscr{L} V(t, x) & =\mathscr{L} h(x)=a x p|x|^{p-2} x+\frac{1}{2} b^{2} x^{2}\left(p(p-2)|x|^{p-4} x^{2}+p|x|^{p-2}\right) \\
& =\left\{a+\frac{b^{2}}{2}(p-1)\right\} p \cdot|x|^{p} \quad \text { for }|x| \geq 1
\end{aligned}
$$

If $a+\frac{b^{2}}{2}(p-1)<0$, then all the conditions in Theorem 3.1 (B) are satisfied. While if $a+\frac{b^{2}}{2}(p-1)>0$, then all the conditions in Theorem 3.2 (B) are satisfied. Thus we are led to the required conclusion by taking $p_{0}=-\frac{2 a}{b_{2}}+1$. Since we may choose $a$ and $b$ arbitrarily, we can give a suitable example for any given $0<p_{0}<\infty$.

In the same manner, we can construct $n$-dimensional examples, as well.

EXAMPLE 3.3. We consider the process $X(t)$ given by

$$
d X(t)=f(t, X(t)) d t+G(t, X(t)) d W(t),
$$

where $f(t, x)$ and $G(t, x)$ satisfy the conditions $\left(\mathrm{A}_{1}\right)$ and $\left(\mathrm{A}_{2}\right)$ in $\S 2$. Let us take a function $V(t, x)$ which is of $C^{2}$-class on $R^{n}$ and is equal to $e^{|x|^{\alpha}}$ for $|x| \geqq 1, \alpha>0$, as a Liapunov function of the process $X(t)$. Then we have

$$
\begin{aligned}
\mathscr{L} V(t, x)= & \left\{\sum_{i} f_{i}(t, x) \alpha x_{i}|x|^{\alpha-2}\right. \\
& \left.+\frac{1}{2} \sum_{i, j} \sigma_{i j}(t, x)\left(\alpha|x|^{2 \alpha-4} x_{i} x_{j}+\alpha(\alpha-2)|x|^{\alpha-4} x_{i} x_{j}+|x|^{\alpha-2} \delta_{i j}\right)\right\} e^{|x|^{\alpha}} \\
\equiv & F(t, x) e^{|x|^{\alpha}} \quad \text { for }|x| \geqq 1 .
\end{aligned}
$$


Therefore, if there exist positive constants $\delta_{1}$ and $\delta_{2}$ such that

$$
F(t, x) \leqq-\delta_{1} \quad \text { for }|x| \geq \delta_{2},
$$

then the condition (iii) in Theorem 3.1 (A) is fulfilled. Since the other conditions in Theorem $3.3(\mathrm{~B})^{\prime}$ are satisfied by $V(t, x)$, it is sufficient to find the conditions assuring (3.14) in order to apply Theorem 3.3 (B)' to the process $X(t)$.

Let us assume that there exist positive constants $\beta_{1}$ and $\beta_{2}$ such that

$$
\lim _{\mid \overline{x \mid \rightarrow \infty}} \frac{|f(t, x)|}{|x|^{\beta_{1}}}>0, \quad \varlimsup_{|x| \rightarrow \infty} \frac{|G(t, x)|}{|x|^{\beta_{2}}}<\infty .
$$

The condition to be assumed next is the following:

$$
(f(t, x), x) \leqq-\delta_{3}|x|^{\beta_{1}+1} \quad \text { for }|x| \geqq \delta_{4},
$$

where $\delta_{3}$ and $\delta_{4}$ are positive constants. (This condition indicates the stability property of the corresponding deterministic system). The function $F(t, x)$ defined above is expressed in the form

$$
\boldsymbol{F}(t, x)=\boldsymbol{F}_{1}(t, x)+\boldsymbol{F}_{2}(t, x)
$$

with $F_{1}(t, x)=\alpha(f(t, x), x)|x|^{\alpha-2}$.

Using the assumptions (3.15) and (3.16), we have

$$
\begin{array}{cc}
F_{1}(t, x) \leqq-\delta_{3} \alpha|x|^{\beta_{1}+\alpha-1} & \text { for }|x| \geqq \delta_{4} \\
\left|F_{2}(t, x)\right| \leqq \delta_{5}(\alpha)|x|^{2 \beta_{1}+2 \alpha-2} & \text { for }|x| \geqq \delta_{4} .
\end{array}
$$

Thus we have two sufficient conditions which assure the inequality (3.14);

(I) Existence of a positive constant $\alpha$ such that $\beta_{1}+\alpha-1 \geqq 0$, $\beta_{1}+\alpha-1>2 \beta_{2}+2 \alpha-2$.

(II) Existence of a positive constant $\alpha$ such that $\beta_{1}+\alpha-1 \geqq 0$, $\beta_{1}+\alpha-1 \geqq 2 \beta_{2}+2 \alpha-2$, and validity of the inequality $\delta_{3} \cdot \alpha>\delta_{5}(\alpha)$.

We may choose a function $\delta(\alpha)=n^{2} c\left\{\alpha^{2}+\alpha(\alpha-2)+1\right\}$ as $\delta_{5}(\alpha)$ in (3.18), where $c$ is the constant in Assumption $\left(\mathrm{A}_{1}\right)$. If we put $\delta_{5}(\alpha)=\delta(\alpha)$ and $\alpha=1-\beta_{1}$, then the inequality $\delta_{3} \cdot \alpha>\delta_{5}(\alpha)$ changes into the following form:

$$
\left(1-\beta_{1}\right) \delta_{3}>n^{2} c\left(1-\beta_{1}+\beta_{1}^{2}\right) .
$$

We can now state the conditions (I) and (II) in terms of $\beta_{i}, i=1,2$, and have 
Proposition 3.1. Let the process $X(t)$ be given by (3.13) and assume that there exist positive constants $\beta_{1}, \beta_{2}, \delta_{3}$ and $\delta_{4}$ which satisfy (3.15) and (3.16).

(i) If $1 \geqq \beta_{1}>\beta_{2}$, then the process $X(t)$ is strongly exponentially $\omega$-th ultimately bounded (therefore it is $p$-th ultimately bounded).

(ii) If $1>\beta_{1}=\beta_{2}$ and the inequality (3.19) is satisfied, then the process $X(t)$ is strongly exponentially $\omega$-th ultimately bounded.

EXAMPLE $3.3^{\prime}$. Let $X(t)$ be the process given by (3.13) in Example 3.3, and take $\log \left(1+|x|^{2}\right)$ as a Liapunov function of the process $X(t)$. In this case we can discuss the ultimate unboundedness in the same manner as Example 3.3, by applying Theorem 3.2, and we can get the alternative proposition to Proposition 3.1. The details are omitted, because our interest is not in the ultimate unboundedness.

\section{§4. Converse theory}

Generally speaking, the main problem of the stability theory is to find a method how to construct Liapunov functions. In approaching this problem, it is most desirable to establish the converse theorems to Theorem 3.1 and Theorem 3.3. We have not succeeded completely, but we can give a partial answer to this problem.

THEOREM 4.1. Let us consider the system (2.1) in §2. We assume, in addition to $\left(\mathrm{A}_{1}\right)$ and $\left(\mathrm{A}_{2}\right)$, that $f(t, x)$ and $G(t, x)$ are of $C^{2}$-class in $x$ and that $f_{x}, f_{x x}, G_{x}$ and $G_{x x}$ are all bounded. Under these assumptions, if the process $X(t)$ given by (2.1) is exponentially $p$-th ultimately bounded for some $p>0$, then there is a function $V(t, x)$ which satisfies the conditions (i), (ii), (iii) and (ii)' in Theorem 3.1 (B).

Proof. Let $h(x)$ be the function defined in Lemma 2.2, and put

$$
V(t, x) \equiv \int_{t}^{t+T} M_{t, x} h(X(u)) d u
$$

where $T$ is a certain positive constant which is to be determined later.

a) Proof of (ii)'. Since $X(t)$ is exponentially $p$-th ultimately bounded, there exists positive constants $c, \alpha$ and $K$ such that

$$
M_{t, x}|X(s)|^{p} \leqq K+c|x|^{p} e^{-\alpha(s-t)} .
$$


Therefore we have

$$
\begin{aligned}
V(t, x) & \leqq \int_{t}^{t+T} M_{t, x}\left(L+|X(u)|^{p}\right) d u \\
& \leqq(L+K) T+c|x|^{p} \int_{t}^{t+T} e^{-\alpha(s-t)} d u \\
& \leqq(L+K) T+\frac{c}{\alpha}|x|^{p},
\end{aligned}
$$

where $L=\sup _{|x| \leqq 1} h(x)$. With $T$ fixed the inequality (4.2) proves (ii)'.

b) Proof of (ii). By Corollary 2.2, it holds that

$$
M_{t, x} h(X(s)) \geqq h(x) e^{-k(s-t)}-C,
$$

where $k$ and $C$ are positive constants. We can choose a positive number $\delta$ so small that $e^{-k \tau}>\frac{1}{2}$ for $0 \leqq \tau \leqq \delta$. Then we have

$$
M_{t, x} h(X(s)) \geqq \frac{1}{2} h(x)-C \quad \text { for } t \leq s \leq t+\delta .
$$

Choose $T$ large than $\delta$ and use (4.4) to obtain

$$
\begin{aligned}
V(t, x) & \geqq \int_{t}^{t+\delta} M_{t, x} h(X(u)) d u+\int_{t+\delta}^{t+T} L^{\prime} d u \\
& \geqq \frac{1}{2} \delta h(x)-C \delta+L^{\prime} T,
\end{aligned}
$$

where $L^{\prime}=\inf _{x} h(x)$. This inequality (4.5) proves (ii).

c) Proof of (i) and (iii). If $h(x)$ is bounded, the differentiability of $M_{t, x} h(X(s))$ in $t$ and $x$ is well-known. (cf. Gikhman-Skorokhod [1]). If $h(x)$ is polynomial, the following lemma follows from Lemma 6.2 in [3] p. 230 with a slight modification. But, in order to prove Theorem 4.3, we shall establish it in the following generalized form.

LEMMA 4.1. Consider a system (2.1) in §2, where we assume, in addition to $\left(\mathrm{A}_{1}\right)$ and $\left(\mathrm{A}_{2}\right)$, that $f(t, x)$ and $G(t, x)$ are of $C^{2}$-class in $x$ and $f_{x}, f_{x x}, G_{x}$ and $G_{x x}$ are all bounded. Let $\varphi(x)$ be a function of $C^{2}$-class defined on $R^{n}$. Then, if $\varphi(x), \varphi_{x}(x)$ and $\varphi_{x x}(x)$ are locally uniformly $r$-th integrable for some $r>1$, the function

$$
u(t, x) \equiv M_{t x} \varphi(X(s)) \quad t<s
$$


is of $C^{1}$-class w.r.t. $t$ and of $C^{2}$-class w.r.t. $x$, and satisfies

$$
\mathscr{L} u(t, x)=0 \quad t<s .
$$

Remark 4.1. We say $\varphi(x)$ is locally uniformly $r$-th integrable (more precisely, $r$-th integrable with respect to the process $X(t))$ if $\varphi(y)$ satisfies

$$
M_{t, x}|\varphi(X(s))|^{r} \leqq C\left(t_{0}, s, D\right)<\infty, \quad \text { for any } t_{0} \leq t \leq s, \quad x \in D \subset R^{n},
$$

where $D$ is any compact subset of $R^{n}$.

We will prove Lemma 4.1 only in the case $n=1$. The proof for the case $n \geq 2$ can be given similarly.

We use the following facts which are easily shown under the assumption of Lemma 4.1 (for details see Khas'minskii [3] pp. 225 227, Gikhman-Skorokhod [1] pp. 403 412) :

1) If we put

$$
Y_{x, \Delta x}^{t}(s) \equiv \frac{1}{\Delta x}\left[X^{t, x+\Delta x}(s)-X^{t, x}(s)\right],
$$

then

$$
M\left[\left(Y_{x, \Delta x}^{t}(s)\right)^{2 m}\right] \leqq e^{k(s-t)},
$$

where $k$ is a constant depending on $\sup \left|f_{x}\right|, \sup \left|G_{x}\right|$ and $m$.

2) If we put

$$
\xi_{x}^{t}(s)=\frac{\partial X^{t, x}}{\partial x}(s) \equiv \lim _{\Delta x \rightarrow 0} X_{x, \Delta x}^{t}(s) \quad \text { (in } L^{2} \text {-sense) }
$$

then $\xi_{x}^{t}(s)$ is well-defined and satisfies

$$
M\left[\left(\xi_{x}^{t}(s)\right)^{2 m}\right] \leqq e^{k(s-t)},
$$

where $k$ is the constant given in 1 ).

3) If we put

$$
\zeta_{x}^{t}(s)=\frac{\partial^{2} X^{t, x}}{\partial x^{2}}(s) \equiv \frac{\partial \xi_{x}^{t}(s)}{\partial x} \quad \text { (in } L^{2} \text {-sense) },
$$

then $\zeta_{x}^{t}(s)$ is well-defined and satisfies

$$
M\left[\left(\zeta_{x}^{t}(s)\right)^{2 m}\right] \leqq c_{1} e^{k(s-t)},
$$


where $c_{1}$ and $k$ are constants depending on $\sup \left|f_{x}\right|, \sup \left|f_{x x}\right|, \sup \left|G_{x}\right|$, $\sup \left|G_{x x}\right|$ and $m$.

Proof of Lemma 4.1 in the case $n=1$. Let $I_{n}(x)$ be a smooth function on $R^{1}$ satisfying

$$
\begin{array}{ll}
I_{n}(x)=1 & |x| \leq n \\
I_{n}(x)=0 & |x| \geqq n+1 \\
\left|I_{n}(x)\right| \leqq 1, & \left|I_{n}^{\prime}(x)\right| \leqq \ell, \quad\left|I_{n}^{\prime \prime}(x)\right| \leq \ell, \quad(\ell \text { constant })
\end{array}
$$

and put $\varphi_{n}(x) \equiv \varphi(x) \cdot I_{n}(x)$. Clearly $\varphi_{n}(x) \rightarrow \varphi(x)$ as $n \rightarrow \infty$, and $\left|\varphi_{n}(x)\right|$ $\leqq \varphi(x)$.

If we define $u_{n}(t, x)$ by

$$
u_{n}(t, x) \equiv M_{t, x} \varphi_{n}(X(s)), \quad t<s(s \text { fixed }),
$$

then we obtain

$$
u_{n}(t, x) \rightarrow u(t, x) \equiv M_{t, x} \varphi(X(s)) \quad(n \rightarrow \infty) .
$$

We see that $u_{n}(t, x)$ is of $C^{1}$-class in $t$ and of $C^{2}$-class in $x$, because $\varphi_{n}(x)$ is bounded. To show that $u(t, x)$ is of $C^{1}$-class in $t$ and of $C^{2}$-class in $x$ it suffices to prove that $u_{n, x}, u_{n, x x}$ and $u_{n, t}$ are uniformly convergent on any compact subset of $(t, x)$-space. Put

$$
u^{*}(t, x) \equiv M\left[\varphi^{\prime}\left(X^{t, x}(s)\right) \xi_{x}^{t}(s)\right] .
$$

This is well-defined because of the $r$-th integrability of $\varphi^{\prime}(x)$ and (4.9). By the definition of $\varphi_{n}(x)$, we have

$$
\varphi_{n}^{\prime}(x) \rightarrow \varphi^{\prime}(x) \quad(n \rightarrow \infty) .
$$

Therefore we obtain

$$
\left.u_{n, x}(t, x)=M\left[\varphi_{n}^{\prime}\left(X^{t, x}(s)\right) \xi_{x}^{t}(s)\right] \rightarrow u^{*}=M\left[\varphi^{\prime}\left(X^{t, x}(s)\right) \xi_{x}^{t}(s)\right)\right],
$$

where we have assumed that the equality

$$
u_{n, x}(t, x)=M\left[\varphi_{n}^{\prime}\left(X^{t, x}(s)\right) \xi_{x}^{t}(s)\right]
$$

holds. (cf. Gikhman-Skorokhood [1]).

We are now ready to prove that the convergence is uniform on compact. 


$$
\begin{aligned}
\left|u^{*}(t, x)-u_{n, x}(t, x)\right| \leqq & M\left[\left|\left\{\varphi^{\prime}-\left(I_{n} \varphi\right)^{\prime}\right\}\left(X^{t, x}(s)\right) \| \xi_{x}^{t}(s)\right|\right] \\
\leqq & \left.M\left[\mid 1-I_{n-1}\left(X^{t, x}(s)\right)\left\|\left(\varphi^{\prime}+\ell \varphi\right)\left(X^{t, x}(s)\right)\right\| \xi_{x}^{t}(s)\right) \mid\right] \\
\leqq & \left\{M\left[\left(1-I_{n-1}\left(X^{t, x}(s)\right)\right)\left|\xi_{x}^{t}(s)\right|\right]^{\prime}\right\}^{1 / r^{\prime}} \\
& \quad \times\left\{M\left[\left(\varphi^{\prime}+\ell \varphi\right)\left(X^{t, x}(s)\right)\right]^{r}\right\}^{1 / r}, \frac{1}{r}+\frac{1}{r^{\prime}}=1, \\
\leqq & C^{\prime}\left(t_{0}, s, D\right)\left\{M\left[\left|1-I_{n-1}\left(X^{t, x}(s)\right) \| \xi_{x}^{t}(s)\right|\right]^{r^{\prime}}\right\}^{1 / r^{\prime}},
\end{aligned}
$$

where the constant $C^{\prime}\left(t_{0}, s, D\right)$ in the last inequality is to be determined by the local uniform integrability of $\varphi(x)$ and $\varphi^{\prime}(x)$. Using the Schwartz inequality, we have

$$
\begin{aligned}
& M\left[\left(1-I_{n-1}\right)\left(X^{t, x}(s)\right)\left|\xi_{x}^{t}(s)\right|\right]^{r^{\prime}} \\
& \quad \leqq\left\{M\left[\left(1-I_{n-1}\right)\left(X^{t, x}(s)\right)\right]^{2 r^{\prime}}\right\}^{1 / 2}\left\{M\left|\xi_{x}^{t}(s)\right|^{2 r^{\prime}}\right\}^{1 / 2} .
\end{aligned}
$$

The Chebyshev inequality proves that

$$
\begin{aligned}
M\left[\left(1-I_{n-1}\right)\left(X^{t, x}(s)\right)\right]^{2 r^{\prime}} & \leqq P\left(\left|X^{t, x}(s)\right| \geqq n-1\right) \leqq \frac{1}{n-1} M\left[\left|X^{t, x}(s)\right|\right] \\
& \leqq \frac{1}{n-1} C^{\prime \prime}\left(t_{0}, s, D\right)
\end{aligned}
$$

Combining (4.13), (4.14), (4.15) and (4.9), we obtain

$$
\begin{aligned}
& \left|u^{*}(t, x)-u_{n, x}(t, x)\right| \\
& \quad \leqq C^{\prime}\left(t_{0}, s, D\right)\left(\frac{1}{n-1} C^{\prime \prime}\left(t_{0}, s, D\right)^{1 / 2 r^{\prime}} e^{\left(1 / 2 r^{\prime}\right) k\left(s-t_{0}\right)}\right) .
\end{aligned}
$$

The inequality (4.16) shows that $u_{n, x}$ converges to $u^{*}(t, x)$ uniformly on $\left[t_{0}, s\right] \times D$. As a consequence, $u_{x}(t, x)$ is continuous and

$$
u_{x}(t, x)=u^{*}(t, x)=M\left[\varphi^{\prime}\left(X^{t, x}(s)\right) \xi_{x}^{t}(s)\right] .
$$

By the same way, using (4.11) in place of (4.9), we can prove that $u_{x x}(t, x)$ is continuous and

$$
\begin{aligned}
u_{x x}(t, x) & =M\left[\varphi^{\prime \prime}\left(X^{t, x}(s)\right) \xi_{x}^{t}(s)\right]+M\left[\varphi^{\prime}\left(X^{t, x}(s)\right) \xi_{x}^{t}(s)\right] \\
& =\lim _{n \rightarrow \infty} u_{n, x x}=\lim _{n \rightarrow \infty} M\left[\varphi_{n}^{\prime \prime}\left(X^{t, x}(s)\right) \xi_{x}^{t}(s)\right]+M\left[\varphi_{n}^{\prime}\left(X^{t, x}(s)\right) \zeta_{x}^{t}(s)\right] .
\end{aligned}
$$

Finally we prove that $u_{t}(t, x)$ exists and that $u(t, x)$ itself satisfies

$$
\mathscr{L} u(t, x)=0 \quad \text { for } t<s .
$$

Here we note that (4.19) holds for $u_{n}(t, x)$. That is 


$$
u_{n, t}(t, x)=-f(t, x) u_{n, x}(t, x)-\frac{1}{2} G^{2}(t, x) u_{n, x x}(t, x) .
$$

The right side converges to

$$
-f(t, x) u_{x}(t, x)-\frac{1}{2} G^{2}(t, x) u_{x x}(t, x)
$$

uniformly on $\left[t_{0}, s\right] \times D$. Thus we have proved that $u_{t}(t, x)$ exists and that

$$
u_{t}(t, x)=\lim _{n \rightarrow \infty} u_{n, t}(t, x)=-f(t, x) u_{x}(t, x)-\frac{1}{2} G^{2}(t, x) u_{x x}(t, x),
$$

which was to be proved.

(Q.E.D.)

We now return to the proof of Theorem 4.1. If we take the above function $h(x)$ as $\varphi(x)$ in Lemma 4.1, then all the assumptions in Lemma 4.1 are satisfied. Thus we see that the function $u(t, x, s)$ given by

$$
u(t, x, s) \equiv M_{t, x} h(X(s))
$$

satisfies

$$
\mathscr{L}_{t, x} u(t, x, s)=0 \quad t \leqq s, x \in R^{n} .
$$

With this, we obtain

$$
\begin{aligned}
\mathscr{L} V(t, x)= & \mathscr{L} \int_{t}^{t+T} u(t, x, s) d s \\
= & \frac{\partial}{\partial t} \int_{t}^{t+T} u(t, x, s) d s+\sum_{i} f_{i}(t, x) \frac{\partial}{\partial x_{i}} \int_{t}^{t+T} u(t, x, s) d s \\
& +\frac{1}{2} \sum_{i, j} \sigma_{i j}(t, x) \frac{\partial^{2}}{\partial x_{i} \partial x_{j}} \int_{t}^{t+T} u(t, x, s) d s \\
= & u(t, x, t+T)-u(t, x, t)+\int_{t}^{t+T} \frac{\partial u}{\partial t}(t, x, s) d s \\
& +\int_{t}^{t+T} \sum_{i} f_{i}(t, x) \frac{\partial}{\partial x_{i}} u(t, x, s) d s \\
& +\int_{\frac{1}{2}} \sum \sigma_{i j}(t, x) \frac{\partial^{2} u}{\partial x_{i} \partial x_{j}}(t, x, s) d s \\
= & u(t, x, t+T)-u(t, x, t)+\int_{t}^{t+T} \mathscr{L}_{t, x} u(t, x, s) d s \\
= & u(t, x, t+T)-u(t, x, t) \\
= & M_{t, x} h(X(t+T))-h(x) .
\end{aligned}
$$


This is bounded above by

$$
L+M_{t, x}|X(t+T)|^{p}-h(x) \leqq L+K+c|x|^{p} e^{-\alpha T}-h(x) \text {. }
$$

Taking $T$ large enough, we obtain from (4.21)

$$
\begin{aligned}
\mathscr{L} V(t, x) & \leqq L+K+\frac{1}{2}|x|^{p}-h(x) \\
& \leqq 2 L+K+\frac{1}{2}-\frac{1}{2}|x|^{p}
\end{aligned}
$$

The inequalities (4.22) and (4.2) show that the condition (iii) is satisfied.

(Q.E.D.)

CoRollary 4.1. Let $X(t)$ satisfy the assumptions of Theorem 4.1, and let $f(t, x)$ and $G(t, x)$ be independent of $t$; denote them by $f(x), G(x)$ respectively. Then

$$
\lim _{|x| \rightarrow \infty} \frac{|f(x)|+|G(x)|}{|x|}>0
$$

- Proof. Since the system is temporally homogeneous, the corresponding Liapunov function given by (4.1) is also independent of $t$. In fact, $V(t, x)$ and $\mathscr{L} V(t, x)$ are of the forms

$$
\begin{gathered}
V(t, x)=V(x)=\int_{0}^{T} M_{x} h(X(\tau)) d \tau \\
\mathscr{L} V(t, x)=\sum_{i} f_{i}(x) \frac{\partial V}{\partial x_{i}}(x)+\frac{1}{2} \sum_{i, j} \sigma_{i j}(x) \frac{\partial^{2} V}{\partial x_{i} \partial x_{j}}(x) .
\end{gathered}
$$

Let us prove

$$
\begin{aligned}
& \left|\frac{\partial V}{\partial x_{i}}(x)\right| \leqq b_{1}|x|^{p-1}+b_{2} \\
& \left|\frac{\partial^{2} V}{\partial x_{i} \partial x_{j}}(x)\right| \leqq b_{3}|x|^{p-2}+b_{4},
\end{aligned}
$$

where $b_{1}, b_{2}, b_{3}$ and $b_{4}$ are positive constants. For simplicity, we consider the case $n=1$. Using (4.17) with $\varphi(x)=h(x)$ and the Schwartz inequality, we obtain 


$$
\begin{aligned}
\left|\frac{\partial V}{\partial x}(x)\right| & =\left|\int_{0}^{T} \frac{\partial}{\partial x} u(0, x, s) d s\right|=\left|\int_{0}^{T} M_{x} h^{\prime}(X(s)) \xi_{x}(s) d s\right| \\
& \leqq \int_{0}^{T}\left\{M_{x} h^{\prime}(X(s))^{2}\right\}^{1 / 2}\left\{M_{x} \xi_{x}(s)^{2}\right\}^{1 / 2} d s .
\end{aligned}
$$

From the inequality (4.9), we have $\left\{M_{x} \xi_{x}(s)^{2}\right\}^{1 / 2} \leqq b$ for $0 \leq s \leq T$, where $\mathrm{b}$ is a constant. Therefore it holds that

$$
\left|\frac{\partial V}{\partial x}(x)\right| \leqq b \int_{0}^{T}\left\{M_{x} h^{\prime 2}(X(s))\right\}^{1 / 2} d s
$$

The obvious inequality

$$
\left|h^{\prime}(x)\right|^{2} \leqq a^{\prime}|x|^{2(p-1)}+b^{\prime}
$$

and Corollary 2.1 prove

$$
M_{x} h^{\prime}(X(s))^{2} \leqq C\left(1+|x|^{p}\right) e^{k T} \quad \text { for } 0 \leq s \leq T .
$$

Combining this inequality with (4.27), we obtain (4.25).

The inequality (4.26) can be proved by the same way, using (4.9), (4.11) and (4.18).

On the other hand $V(x)$ satisfies the conditions in Theorem 3.1 (B) by a consequence of Theorem 4.1. Thus the following inequality must be satisfied:

$$
\mathscr{L} V(x) \leqq-b_{5}|x|^{p}+\beta .
$$

Observing (4.24), (4.25), (4.26) and (4.28), we see that the conclusion of Corollary is a necessary condition in order that the conditions (4.25), (4.26) and (4.28) are all satisfied.

CoRollary 4.2. Let $X(t)$ be the process which satisfies all the assumptions in Theorem 4.1 for $p=4 / 3$ and the following condition:

$$
|G(t, x)| \leqq c_{11}\left(1+|x|^{r}\right),
$$

where $0 \leqq r<1 / 3$. Then the process $X(t)$ is strongly exponentially $\omega$-th ultimately bounded.

Proof. Let $V(t, x)$ be the Liapunov function which is given by (4.1) with $p=4 / 3$ in the proof of Theorem 4.1. We will show that the function $W(t, x)=e^{V(t, x)}$ satisfies all the conditions in Theorem $3.3(B)^{\prime}$. It 
is clear that $W(t, x)$ satisfies the conditions (i), (iv) and (iv)". Taking derivatives of $W(t, x)$, we have

$$
\mathscr{L} W(t, x)=e^{V(t, x)}\left(\mathscr{L} V(t, x)+\frac{1}{2} \sum_{i, j} \sigma_{i j}(t, x) \frac{\partial V}{\partial x_{i}}(t, x) \frac{\partial V}{\partial x_{j}}(t, x)\right) .
$$

As $V(t, x)$ satisfies the condition (iii) in Theorem 3.1, there are two constants $c_{12}$ and $\beta$ such that

$$
\mathscr{L} V(t, x) \leqq-c_{12}|x|^{4 / 3}+\beta
$$

Therefore we have

$$
\begin{aligned}
\mathscr{L} W(t, x) & \leqq W(t, x) \\
& \times\left(-c_{12}|x|^{4 / 3}+\beta+\frac{1}{2} \sum_{i, j}\left|\sigma_{i j}(t, x) \frac{\partial V}{\partial x_{i}}(t, x) \frac{\partial V}{\partial x_{j}}(t, x)\right|\right) .
\end{aligned}
$$

Using (4.25) in the proof of Corollary 4.1 and (4.29), the inequality (4.30) is changed into

$$
\begin{aligned}
\mathscr{L} W(t, x) & \leqq W(t, x)\left\{-c_{12}|x|^{4 / 3}+\beta+\frac{1}{2} \sum_{i, j}\left|\sigma_{i, j}\right|\left(b_{1}|x|^{1 / 3}+b_{2}\right)^{2}\right\} \\
& \leqq W(t, x)\left\{-c_{12}|x|^{4 / 3}+\beta+\frac{1}{2} n^{2} c_{11}^{2}\left(1+|x|^{r}\right)^{2}\left(b_{1}|x|^{1 / 3}+b_{2}\right)^{2}\right\} .
\end{aligned}
$$

Since $r$ is smaller than $1 / 3$, we obtain from (4.31)

$$
\mathscr{L} W(t, x) \leqq W(t, x)\left\{-\frac{1}{2} c_{12}|x|^{4 / 3}+\beta^{\prime}\right\},
$$

where $\beta^{\prime}$ is a constant. The inequality (4.32) is equivalent to the condition (iii). Now it is easy to check that $W(t, x)$ satisfies the other conditions in Theorem $3.3(B)^{\prime}$.

(Q.E.D.)

THEOREM 4.2. Consider a system (2.1) in §2. We assume that $f(t, x)$ and $G(t, x)$ are of $C^{2}$-class in $x$ and $f_{x}$ and $f_{x x}$ are bounded and that $G(t, x)$ has a compact carrier as a function of $x$. If the process $X(t)$ governed by (2.1) is p-th ultimately bounded, then there exists a Liapunov function $V(t, x)$ which satisfies the conditions (i), (ii) and (iii) in Theorem 3.1 (A).

Proof. Since the process $X(t)$ is $p$-th ultimately bounded, there is a constant $K$ such that 


$$
\varlimsup_{s \rightarrow \infty} M_{t, x}|x(s)|^{p} \leqq K \quad \text { for any }(t, x) \text {. }
$$

Taking two constants $K_{1}$ and $K_{2}$ with $K<K_{1}<K_{2}$, we define a smooth function $G(r)$ on $R^{1}$ by $G(r)=r-K_{2}+1$, for $r \geqq K_{2}, G(r)=0$, for $r \leqq K_{1}, 0 \leqq G(r) \leqq 1$, for $K_{1}<r<K_{2}$. Set

$$
V(t, x) \equiv \int_{t}^{\infty} G(u(t, x, s)) e^{\lambda(\tau-t)} d \tau, \quad \lambda>0,
$$

where $u(t, x, s)=M_{t, x} h(X(s))$.

a) Proof of (ii). We can carry out the proof by the same way as that of b) in Theorem 4.1.

b) Proof of (i) and (iii). The smoothness of $V(t, x)$ is trivial. To find the expression for $\mathscr{L} V(t, x)$ we proceed as follows.

$$
\begin{aligned}
\frac{\partial V}{\partial t}(t, x)= & -G(u(t, x, t))+\int_{t}^{\infty} G^{\prime}(u(t, x, \tau)) u_{t}(t, x, \tau) e^{\lambda(\tau-t)} d \tau \\
& -\lambda \int_{t}^{\infty} G(u(t, x, \tau)) e^{\lambda(\tau-t)} d \tau \\
= & -|x|^{p}+K_{2}-1+\int_{t}^{\infty} G^{\prime}(u(t, x, \tau)) u_{t}(t, x, \tau) e^{\lambda(\tau-t)} d \tau-\lambda V(t, x) \\
\frac{\partial V}{\partial x_{i}}(t, x)= & \int_{t}^{\infty} G^{\prime}(u(t, x, \tau)) u_{x_{i}}(t, x, \tau) e^{\lambda(\tau-s)} d \tau . \\
\frac{\partial^{2} V}{\partial x_{i} \partial x_{j}}(t, x)= & \int_{t}^{\infty} G^{\prime \prime}(u(t, x, \tau)) u_{x_{i}}(t, x, \tau) u_{x_{j}}(t, x, \tau) e^{\lambda(\tau-t)} d \tau \\
& +\int_{t}^{\infty} G^{\prime}(u(t, x, \tau)) u_{x_{i} x_{j}}(t, x, \tau) e^{\lambda(\tau-t)} d \tau .
\end{aligned}
$$

Therefore we have, for $|x| \geq K_{2}$,

$$
\begin{gathered}
\mathscr{L} V(t, x)=-|x|^{p}+K_{2}-1-\lambda V(t, x)+\int_{t}^{\infty} G^{\prime}(u(t, x, \tau)) \mathscr{L}_{t, x} u(t, x, \tau) e^{\lambda(\tau-t)} d \tau \\
+\frac{1}{2} \sum_{i, j} \sigma_{i j}(t, x) \int_{t}^{\infty} G^{\prime \prime}(u(t, x, \tau)) u_{x_{i}}(t, x, \tau) u_{x_{j}}(t, x, \tau) e^{\lambda(\tau-t)} d \tau \\
=-|x|^{p}-\lambda V(t, x)+K_{2}-1+\frac{1}{2} \sum_{i, j} \sigma_{i j} \\
\times \int_{t}^{\infty} G^{\prime \prime}(u(t, x, \tau)) u_{x_{i}}(t, x, \tau) u_{x_{j}}(t, x, \tau) e^{\lambda(\tau-t)} d \tau,
\end{gathered}
$$

where we have used $\mathscr{L}_{t, x} u(t, x, \tau)=0$. By the assumption that $G(t, x)$ has a compact carrier, 


$$
\sigma_{i j}(t, x)=0, \quad \text { for }|x| \geqq K_{3} \text {. }
$$

From (4.33) and (4.34) we obtain

$$
\mathscr{L} V(t, x) \leqq-\lambda V(t, x)-|x|^{p}+K_{2}-1 \quad \text { for }|x| \geqq \max \left\{K_{2}, K_{3}\right\}
$$

Thus the condition (iii) has been verified.

THEOREM 4.3. Consider a system (2.1) in $\S 2$, and assume that the coefficients $f(t, x)$ and $G(t, x)$ satisfy the same assumptions as those of Theorem 4.1. If the process $X(t)$ governed by (2.1) is strongly exponentially $\omega$-th ultimately bounded, then there exists a Liapunov function $V(t, x)$ which satisfies the conditions (i), (iii), (iv) and (iv)' in Theorem 3.3 (B).

Proof. From the definition of strongly exponentially $\omega$-th ultimate boundedness there is an associated function $\varphi(x)=e^{a|x|^{b}}$ satisfying

$$
M_{t, x} \varphi(X(s)) \leqq C \cdot \varphi(x) e^{-\alpha(s-t)}+K
$$

We introduce a $C^{2}$-class function $\bar{\varphi}(x)$ such that

$$
\bar{\varphi}(x)=\varphi(x) \quad \text { for }|x| \geqq 1 .
$$

The inequality (4.35) still holds for $\bar{\varphi}(x)$ by replacing, if necessary, $K$ with a new constant $K^{\prime}$ :

$$
M_{t, x} \bar{\varphi}(X(s)) \leqq C \cdot \bar{\varphi}(x) e^{-\alpha(s-t)}+K^{\prime} .
$$

a) Put

$$
\psi(x) \equiv \sqrt{\bar{\varphi}(x)}
$$

We will first show that $\psi(x)$ satisfies the assumption in Lemma 4.1. It is clear that $\psi(x)$ is locally uniformly square integrable by (4.36) and (4.37). Take a constant $r_{1}$ such that $2>r_{1}^{2}>1$. Then we have

$$
\begin{array}{ll}
\left|\psi_{x_{i}}(x)\right| \leqq \delta & \text { for }|x| \leq 1, \\
\left|\psi_{x_{1}}(x)\right| \leqq|\psi(x)|\left(\frac{1}{2} a b|x|^{b-1}+\delta\right) & \text { for }|x| \geqq 1,
\end{array}
$$

where $\delta$ is a constant. The Hölder inequality proves that 


$$
\begin{aligned}
M_{t, x}|(X(s))|^{r_{1}} \leqq & M_{t, x}|\psi(X(s))|^{r_{1}}\left(\frac{1}{2} a b|X(s)|^{b-1}+\delta\right)^{r_{1}}+\delta^{r_{1}} \\
\leqq & \left\{M_{t, x}|\psi(X(s))|^{r_{1}^{2}}\right\}^{1 / r_{1}}\left\{M_{t, x}\left(\frac{1}{2} a b|X(s)|^{b-1}+\delta\right)^{r_{1} r_{1}^{\prime}}\right\}^{1 / r_{1}^{\prime}} \\
& \quad+\delta^{r_{1}}, \quad \frac{1}{r_{1}}+\frac{1}{r_{1}^{\prime}}=1 .
\end{aligned}
$$

The inequality (4.39) means the locally uniform $r_{1}$-th integrability of $\psi_{x_{i}}(x)$ if $b \geqq 1$, because $\psi(x)$ is locally uniformly $r_{1}^{2}$-th integrable and $|x|^{b-1}$ is locally uniformly $r_{1} r_{1}^{\prime}$-th integrable by Corollary 2.1. While, if $b<1$, we have $\left|\psi_{x_{i}}(x)\right| \leqq|\psi(x)| \delta^{\prime}$ with some constant $\delta^{\prime}>0$ by (4.38), so that $\psi_{x_{i}}(x)$ is locally uniformly $r_{1}$-th integrable.

We take a constant $r_{2}$ such that $1<r_{2}^{2}<r_{1}<2$. Then

$$
\begin{gathered}
\left|\psi_{x_{i} x_{j}}(x)\right| \leqq \delta^{\prime \prime} \quad \text { for }|x|<1, \\
\left|\psi_{x_{i} x_{j}}(x)\right| \leqq \\
\quad|\psi(x)|\left(\frac{1}{4} a^{2} b^{2}|x|^{2(b-1)}+\delta^{\prime \prime}\right) \\
+|\psi(x)|\left(\frac{1}{2} a b(b-1)|x|^{b-2}+\delta^{\prime \prime}\right),
\end{gathered}
$$

where $\delta^{\prime \prime}$ is a positive constant.

Thus the locally uniform $r_{2}$-th integrability of $\psi_{x_{i} x_{j}}(x)$ can be proved as before.

b) Put

$$
u(t, x, s) \equiv M_{t, x} \psi(X(s))
$$

then, by the use of Lemma 4.1, we have

$$
\mathscr{L}_{t, x} u(t, x, s)=0 \quad \text { for } t<s .
$$

By using (4.36), (4.37) and the Schwartz inequality we obtain

$$
\begin{aligned}
u(t, x, s) & \leqq\left[M_{t, x} \bar{\varphi}(X(s))\right]^{1 / 2} \\
& \leqq\left(C \bar{\varphi}(x) e^{-\alpha(s-t)}+K^{\prime}\right)^{1 / 2} \leqq C^{1 / 2} \psi(x) e^{(-1 / 2) \alpha(s-t)}+K^{\prime 1 / 2}
\end{aligned}
$$

c) Put

$$
V(t, x)=\int_{t}^{t+T} u(t, x, \tau) d \tau,
$$

where $T$ is a constant which is to be determined later. We will show that the $V(t, x)$ has the desired properties. The Jessen inequality and Corollary 2.2 show that 


$$
\begin{aligned}
u(t, x, \tau) & =M_{t, x} \psi(X(\tau)) \geqq M_{t, x}\left[e^{\left\{(1 / 2) a|X(\tau)|^{b}-d_{1}\right\}}\right] \\
& \geqq e^{\left\{(1 / 2) a M_{t, x}|X(\tau)|^{b}-d_{1}\right\}} \\
& \geqq e^{\left\{(1 / 2) a|x|^{b}-k(\tau-t)-d_{2}\right\}}=c_{13} e^{(1 / 2) a|x|^{b} e-k(\tau-t)} \\
& \geqq c_{14} e^{d_{4} a|x|^{b}} \quad \text { for } t \leq \tau \leq t+T,
\end{aligned}
$$

where $d_{1}$ and $d_{2}$ are constants and $c_{13}=e^{-d_{2}}, d_{4}=(1 / 2) e^{-k T}$. Thus the condition (iv) is satisfied. As for the condition (iv)' we proceed as follows :

$$
\begin{aligned}
V(t, x) & \leqq c^{1 / 2} \psi(x) \int_{t}^{t+T} e^{(-1 / 2) \alpha(\tau-t)} d \tau+K^{1 / 2} T \\
& \leqq c_{15} e^{(1 / 2) a|x|^{b}}+c_{16}, \quad\left(c_{15}>0, c_{16}>0\right) .
\end{aligned}
$$

The properties that $V(t, x)$ is of $C^{1}$-class in $t$ and of $C^{2}$-class in $x$ are inherited from $u(t, x, \tau)$.

We finally prove that $V(t, x)$ satisfies the condition (iii). By the use of (4.41) as in (4.20),

$$
\begin{aligned}
\mathscr{L} V(t, x) & =u(t, x, t+T)-u(t, x, t) \\
& \leqq c^{1 / 2} \psi(x) e^{(-1 / 2) \alpha T}+K^{\prime 1 / 2}-\psi(x) \\
& \leqq-c_{17} e^{(1 / 2) a|x|^{b}}+K^{\prime 1 / 2}
\end{aligned}
$$

Here we choose the constant $T$, which has been kept undefinite, so large that the last inequality holds with positive constant $c_{17}$. The inequalities (4.43) and (4.44) assure the condition (iii).

(Q.E.D.)

\section{§5. Recurrence property.}

W. M. Wonham ([5]) has discussed on the positive recurrence property of a temporary homogeneous system given by a stochastic differential equation by means of Liapunov functions. He studied the system given by

$$
d X(t)=f(X(t)) d t+G(X(t)) d W(t),
$$

where the coefficients $f(x)$ and $G(x)$ satisfy Lipchitz condition and $G(x)$ is non-degenerate, i.e. $G(x) G(x)^{*}$ is a strictly positive definite matrix. One of his results is that the existence of a Liapunov function $V(x)$, which satisfies the condition $\mathscr{L} V(x) \leqq-1$ for $|x|$ large enough, is necessary and sufficient in order that the system is positive recurrent (cf. Lemma 2 and Theorem 2 in [5]). 
Our results in $\S 4$ assert the existence of Liapunov functions. Combining Theorem 4.1 and the Wonham's result, we conclude that if the system governed by (5.1) is non-degenerate and if it is exponentially $p$-th ultimately bounded for some $p>0$, then it is positive recurrent. If we replace Theorem 4.1 with Theorem 4.3 , we have again a similar conclusion.

We note that Ex. 3.2 shows that the system which is exponentially $p$-th ultimately unbounded may be positive recurrent. From this fact we know that, in the case where the system is temporary homogeneous and non-degenerate, the concept of exponentially $p$-th ultimate boundedness expresses stronger stability than positive recurrence.

For further development we should investigate the recurrence properties of systems even if the system is not temporary homogeneous or may not be non-degenerate. Either case is interesting in many aspects.

Let us first introduce definitions of recurrence property which are suitable for possibly degenerate cases.

DEFINITION 5.1. A process $X(t), t \geqq 0$, is said to be weakly recurrent if there exists a constant $K$ such that

$$
P_{t, x\{}\{\omega ;|X(t+s)| \leq K \text { for some } s \geq 0\}=1 \text { for any }(t, x),
$$

where $P_{t, x}$ stands for the conditional probability under the initial condition $X(t)=x$. The set $\{x ;|x| \leq K\}$ is said to be a recurrent region.

DEFINITION 5.2. A process $X(t), t \geqq 0$, is said to be weakly positive recurrent if there exists a constant $K$ and the following condition is satisfied for the first hitting time $\tau(\omega)$ to the region $\{x ;|x| \leq K\}$;

$$
M_{t, x} \tau(\omega)<\infty \quad \text { for any }(t, x) .
$$

THEOREM 5.1. The process $X(t)$ defined by (2.1) is weakly recurrent if it is p-th ultimately bounded for some $p>0$.

Before we prove this theorem, let us prepare a lemma.

Lemma 5.1. Let $X(t)$ be a Markov process. If there are a positive Borel measurable function $\rho(t, x)$ defined on $[0, \infty) \times R^{n}$ and two positive constants $K$ and $\alpha$ such that

$$
P_{t, x}\{\omega ;|X(t+\rho(t, x), \omega)| \leqq K\} \geqq \alpha>0 \quad \text { for any }(t, x),
$$


then the process $X(t)$ is weakly recurrent and $\{x ;|x| \leq K\}$ is the recurrent region.

Proof. For a fixed $(t, x)$, let us define subsets $\Omega_{i}, i=1,2, \cdots, \infty$, of $\Omega$ as follows;

$$
\begin{aligned}
\Omega_{1} & =\{\omega \in \Omega ;|X(t+\rho(t, x), \omega)|>K\}, \\
\Omega_{2} & =\left\{\omega \in \Omega_{1} ;|X(t+\rho(t, x)+\rho(t+\rho(t, x), X(t+\rho(t, x))), \omega)|>K\right\}, \\
& \ldots \\
\Omega_{\infty} & =\bigcap_{i \geqq 1} \Omega_{i} .
\end{aligned}
$$

Since $\{\omega ;|X(t+s, \omega)|>K$, for any $s \geqq 0\} \subset \Omega_{\infty}$, it is sufficient to prove $P_{t, x}\left(\Omega_{\infty}\right)=0$. Form the assumption of Lemma 5.1,

$$
P_{t, x}\left(\Omega_{1}\right) \leqq 1-\alpha<1
$$

By the use of the Markov property and the measurability of $\rho(t, x)$, we have

$$
\begin{aligned}
P_{t, x}\left(\Omega_{2}\right) & =M_{t, x}\left\{\chi_{\Omega_{1}}(\omega) P_{t+\rho(t, x), X(t+\rho(t, x), \omega)}\left(\Omega^{\prime}\right)\right\} \\
\Omega^{\prime} & \equiv\{\omega ;|X(t+\rho(t, x)+\rho(t+\rho(t, x), X(t+\rho(t, x))))|>K\} .
\end{aligned}
$$

The inequality (5.2) is true for any $(t, x)$, i.e.

$$
\begin{aligned}
& P_{t+\rho(t, x), X(t+\rho(t, x), \omega)}\{\omega ;|X(t+\rho(t, x)+\rho(t+\rho(t, x), X(t+\rho(t, x))))|>K\} \\
& \quad \leqq 1-\alpha .
\end{aligned}
$$

From (5.3) and (5.4), we get

$$
P_{t, x}\left(\Omega_{2}\right) \leqq(1-\alpha)^{2}
$$

In the same manner,

$$
P_{t, x}\left(\Omega_{i}\right) \leqq(1-\alpha)^{i} \rightarrow 0 \quad \text { as } i \rightarrow \infty .
$$

This proves $P_{t, x}\left(\Omega_{\infty}\right)=0$.

Proof of Theorem 5.1. It is clear that $M_{t, x}|X(s)|^{p}$ is continuous in $(t, x)$ for a fixed $s$ and is continuous in $s$ for a fixed $(t, x)$. (cf. Lemma 4.1, where it has been proved that if the coefficients of (2.1) are smooth, then $M_{t, x}|X(s)|^{p}$ is smooth.) From this fact we are able to define a Borel function $\rho(t, x)$ such that

$$
M_{t, x}|X(t+\rho(t, x))|^{p} \leqq K_{1}^{p} \equiv(1+\varepsilon) K^{\prime},
$$


where $K^{\prime}$ is the constant determining the ultimate boundedness of $X(t)$ and $\varepsilon$ is a positive constant.

We will next determine constants $K$ and $\alpha$ so that the inequality (5.1) is satisfied. Put $K=K_{1}+\varepsilon$. Then the following inequality is valid by (5.5),

$$
\begin{gathered}
P_{t, x}\{\omega ;|X(t+\rho(t, x))|>K\}=P_{t, x}\left\{\omega ;|X(t+\rho(t, x))|^{p}>K^{p}\right\} \\
\leqq \frac{1}{K^{p}} M_{t, x}|X(t+\rho(t, x))|^{p} \leqq \frac{K_{1}^{p}}{K^{p}} \equiv 1-\alpha<1 .
\end{gathered}
$$

This proves that the process $X(t)$ is weakly recurrent.

In the case of exponential type, more precise result is obtained.

THEOREM 5.2. The process $X(t)$ defined by (2.1) is weakly positive recurrent if it is exponentially $p$-th ultimately bounded for some $p>1$.

This theorem is obtained as a corollary of the following lemma.

LEMMA 5.2. Let $X(t)$ be a Markov process. If there exist a positive non-decreasing function $W(r)$ defined on $[0, \infty)$ and two positive constants $K$ and $p$ such that

$$
M_{t, x}|X(t+s)|^{p} \leqq K^{p} \quad \text { for any }(t, x) \text { and } s \geqq W(|x|),
$$

and

$$
\sum_{\ell=1}^{\infty} \frac{1}{\ell^{p}} W((\ell+1) N)<\infty \quad \text { for any } N \geqq 0 \text {, }
$$

then $X(t)$ is weakly positive recurrent.

Proof. Let us first notice the following inequality

$$
\begin{aligned}
& P_{t, x}\{\omega ;|X(t+W(|x|))| \geqq k K\} \\
& \quad \leqq \frac{1}{(k K)^{p}} M_{t, x}|X(t+W(|x|))|^{p} \leqq \frac{1}{k^{p}}
\end{aligned}
$$

Set $K^{\prime}=(1+\varepsilon) K$, where $\varepsilon$ is a positive constant to be determined later. We will show that $X(t)$ is a weakly positive recurrent process with the recurrent region $E_{0}=\left\{x ;|x| \leq K^{\prime}\right\}$.

Put $E_{\ell}=\left\{x ; \ell K^{\prime}<|x| \leqq(\ell+1) K^{\prime}\right\}$ and $W^{\prime}(\ell)=W\left(\ell K^{\prime}\right), \ell=1,2, \cdots$, and fix the starting point $(t, x)$. We define a sequence $\left\{x_{m}(\omega)\right\}_{m=1,2, \ldots}$, for almost all $\omega\left(P_{t, x}\right)$ as follows; 


$$
\begin{aligned}
x_{0}(\omega) & \equiv x, \\
x_{1}(\omega) & =X(t+W(|x|), \omega), \\
x_{2}(\omega) & =X\left(t+W(|x|)+W^{\prime}(\ell+1), \omega\right) \quad \text { if } x_{1}(\omega) \in E_{\ell}, \\
\ldots & \\
x_{m}(\omega) & =X\left(t+W(|x|)+W^{\prime}\left(\ell_{1}+1\right)+\cdots+W^{\prime}\left(\ell_{m-1}+1\right), \omega\right) \\
& \text { if } x_{1}(\omega) \in E_{\ell_{1}}, \cdots, x_{m-1} \in E_{\ell_{m-1}} .
\end{aligned}
$$

Then $\Omega$ is represented as

$$
\Omega=\sum_{m=1}^{\infty} \Omega_{m} \bmod .0\left(P_{t, x}\right)
$$

where $\Omega_{m}=\left\{\omega \in \Omega ; x_{1}(\omega) \notin E_{0}, \cdots, x_{m-1}(\omega) \notin E_{0}, x_{m}(\omega) \in E_{0}\right\}$. This is true because $X(t)$ is weakly recurrent by Lemma 5.1 and $E_{0}$ is a current region for any $\varepsilon>0$. Let us further devide $\Omega_{m}$ as follows;

$$
\Omega_{m}=\sum_{\ell_{1}, \ldots, \ell_{m-1} \geqq 1} \Omega_{m, \ell_{1}, \cdots, \ell_{m-1}},
$$

where $\Omega_{m, \ell_{1}, \cdots, \ell_{m-1}}=\left\{\omega ; x_{1}(\omega) \in E_{\ell_{1}}, \cdots, x_{m-1}(\omega) \in E_{\ell_{m-1}}, x_{m}(\omega) \in E_{0}\right\}$.

Define $\tau(\omega)$ as the hitting time to $E_{0}$, then the following inequality is satisfied

$$
\begin{aligned}
\tau(\omega) & \leqq W(|x|) \quad \text { on } \Omega_{1} \\
& \leqq W(|x|)+W^{\prime}\left(\ell_{1}+1\right) \quad \text { on } \Omega_{1, \ell_{1}}, \\
& \cdots \\
& \leqq W(|x|)+W^{\prime}\left(\ell_{1}+1\right)+\cdots+W^{\prime}\left(\ell_{m-1}+1\right) \quad \text { on } \Omega_{m, \ell_{1}, \cdots, \ell_{m-1}} .
\end{aligned}
$$

Therefore we have

$$
\begin{aligned}
& \mathbf{M}_{t, x}[\tau(\omega)] \\
& \leqq \sum_{m, \ell_{1}, \cdots, \ell_{m-1} \geqq 1} P_{t, x}\left(\Omega_{m, \ell_{1}, \cdots, \ell_{m-1}}\right)\left[W(|x|)+W^{\prime}\left(\ell_{1}+1\right)+\cdots+W^{\prime}\left(\ell_{m-1}+1\right)\right] .
\end{aligned}
$$

To evaluate the right hand side of the above, we proceed as follows.

$$
P_{t, x}\left(\Omega_{m, \ell_{1}, \cdots, \ell_{m-1}}\right)<\frac{1}{(1+\varepsilon)^{p(m-1)}} \frac{1}{\left(\ell_{1}\right)^{p}} \cdots \frac{1}{\left(\ell_{m-1}\right)^{p}} .
$$

This inequality is proved inductively by the use of 


$$
\begin{aligned}
& P_{t, x}\left\{\Omega_{m+1, \ell_{1}, \cdots, \ell_{m}}\right\} \leqq P_{t, x}\left\{\sum_{\ell \geqq \ell_{m}} \Omega_{m+1, \ell_{1}, \cdots, \ell_{m-1}, \ell}\right\} \\
&= M_{t, x}\left\{\chi_{\Omega_{m}, \ell_{1}}, \cdots, \ell_{m-1}\right. \\
&\left.\left.\quad\left(s=W(|x|)+W_{s, X_{m}(\omega)}\left\{\omega ; x_{m+1}(\omega)>\ell_{m} K^{\prime}\right\}\right\}, 1\right)+\cdots+W^{\prime}\left(\ell_{m-1}+1\right)\right) \\
&=M_{t, x}\left\{\chi_{\Omega_{m, \ell_{1}}, \cdots, \ell_{m-1}} P_{s, X_{m}(\omega)}\left\{\omega ; x_{m+1}(\omega)>\ell_{m}(1+\varepsilon) K\right\}\right\} \\
& \leqq \\
& \leqq
\end{aligned}
$$

and

$$
P_{t, x}\left(\Omega_{1}\right)<1 \text {. }
$$

Using (5.10) and (5.11), we get

$$
\begin{aligned}
& M_{t, x}[\tau(\omega)] \\
& \leqq W(|x|)+\sum_{m=2}^{\infty} \frac{1}{(1+\varepsilon)^{p(m-1)}} \\
& \quad \times \sum_{\ell_{1}, \ldots, \ell_{m-1} \geqq 1} \frac{W(|x|)+W^{\prime}\left(\ell_{1}+1\right)+\cdots+W^{\prime}\left(\ell_{m-1}+1\right)}{\left(\ell_{1}\right)^{p} \cdots\left(\ell_{m-1}\right)^{p}} \\
& =W(|x|)+\sum_{m=2}^{\infty} \frac{1}{(1+\varepsilon)^{p(m-1)}}\left\{W(|x|) \sum_{\ell_{1}, \cdots, \ell_{m-1} \geqq 1} \frac{1}{\left(\ell_{1}\right)^{p} \cdots\left(\ell_{m-1}\right)^{p}}\right. \\
& =W(|x|)+\sum_{m=2}^{\infty} \frac{1}{(1+\varepsilon)^{p(m-1)}}\left\{W(|x|) A^{m+1}+(m-1) \sum_{\ell_{1}, \ldots, \ell_{m-1} \geqq 1} \frac{W^{\prime}\left(\ell_{1}+1\right)}{\left(\ell_{1}\right)^{p} \cdots\left(\ell_{m-1}\right)^{p}}\right\} \\
& \left.=W(|x|)+A^{m-2} B\right\}
\end{aligned}
$$

where $A=\sum_{\ell=1}^{\infty} \frac{1}{\ell^{p}}$ and $B=\sum_{\ell=1}^{\infty} \frac{1}{\ell^{p}} W^{\prime}(\ell+1)$ which converge by the assumption (5.8). The right side of (5.12) converges if we choose $\varepsilon$ large enough.

Proof of Theorem 5.2. Let $K_{1}$ be the constant determining the ultimate boundedness of $X(t)$ and let $K=K_{1}+1$. As we mentioned after Theorem 3.1 (cf. Remark 3.2), the condition (5.7) is fulfilled putting $W(r)=d_{1} \log (1+r)+d_{2}$. The condition (5.8) is valid since $p>1$ and $W(r)$ is a logarithmic function. Therefore Lemma 5.2 can be applied to this process and we get the conclusion.

(Q.E.D.) 


\section{BIBLIOGRAPHY}

[1] I. G. Gikhman-A. V. Skorokhod, Introduction to the Theory of Random Processes, 1965.

[2] M. B. Nevel'son and P. Z. Khas'minskii, Stability of Stochastic Systems, Problemy Peredachi Informatsii, vol. 2, No. 3, pp. 76-91, 1966.

[ 3 ] Р. 3. Хасьминский; Чстойчивость систем дифференциальных уравнений прп случайных возмущениях их параметров, 1969.

[4] M. Zakai, On the Ultimate Boundedness of Moments Associated with Solutions of Stochastic Differential Equations, SIAM J. Control, vol.5, No. 4, 1967, pp. 588-593.

[ 5 ] W. M. Wonham, Liapunov Criteria for Weak Stochastic Stability, J. of Diff. Eq., 2, pp. 195-207, 1966.

[ 6 ] N. N. Krasovskii, Stability of Motion, Stanford Univ. Press, 1963.

Nagoya University 